\title{
On-site Robotic Assembly of Double-curved Self-supporting Structures
}

\author{
- Inés Ariza \\ MIT, USA \\ iariza@mit.edu \\ - Merav Gazit \\ MIT, USA \\ mgazit@mit.edu
}

\begin{abstract}
Robotic assembly of architectural structures has been an area of research for a few decades. Yet, current methods impose a large number of constraints on the geometry of those structures. In this paper we introduce a method for robotic assembly that enables the construction of double curved self-supporting structures. Latest research challenges have focused on the assembly of sophisticated brick structures and on sensor feedback systems for handling accuracy. We propose an alternative strategy to tackle tolerance handling in complex structures that rely on geometry. The intelligence of the system lies in two main aspects: a subdivision technique that incorporates the robot's constraints as well as the structural equilibrium of the structure during each step of assembly, in order to omit the use of scaffolding; and a match between geometric information and the robot's movements in a robot programming environment. As a proof of concept, we fabricated a portion of a full-scale double-curved structure. The structure was assembled without scaffolding by a portable KUKA KR10 on a randomly picked site. This project aims to demonstrate an easy and simple method for robotic assembly that enables the realization of digitally generated complex geometries as concrete complex structures.
\end{abstract}

Keywords: Robotic Assembly, Self-supporting Structure, On-site Assembly, Double Curvature, Construction Tolerances

\section{Introduction}

The introduction of digital tools and associative modeling into design in the last few decades allowed architects and designers to create complex geometries relatively easily. Yet, when realizing these complex structures in the 'real world', that easiness fades away, the process becomes tedious and ad-hoc, and often demands some painful compromises in the final outcome.

In this paper we propose to rethink the designconstruction workflow by providing geometric information of the design model for an on-site robotic arm that performs the assembly process. The robotic arm is then an integrated part of the design process from its early stages, which in turn accounts for a more continuous and smooth designconstruction workflow.

In many industries, and for many years now, industrial robots have been crucial components in complex assembly lines (e.g. the car industry) (Carlsson, 1989). As the ability of the designer to easily generate complex structures increases, the demand for an analogous assembly technology in the building industry is evident. The gap between cutting-edge digital design methods and current on-site construction methods is quite substantial, as the greater part of the building industry still uses standard tools. The automation of the assembly process will decrease the gap between design and construction processes, and between the initial architectural model and the final outcome.

In recent years we have seen multiple projects that tackle the issue of robotic assembly in architecture. The majority of them involve vertical brick stacking, where a minor change in the orientation of each brick allows the creation of non-standard structures (Figure 1). This strategy, though impressive and novel, imposes a great number of constrains on the geometry and on the final outcome. We propose an alternative method for assembly that enables the construction of double curved self-supporting structures. (For the purpose of this paper, we will call these structures 'complex structures' from now on.) 


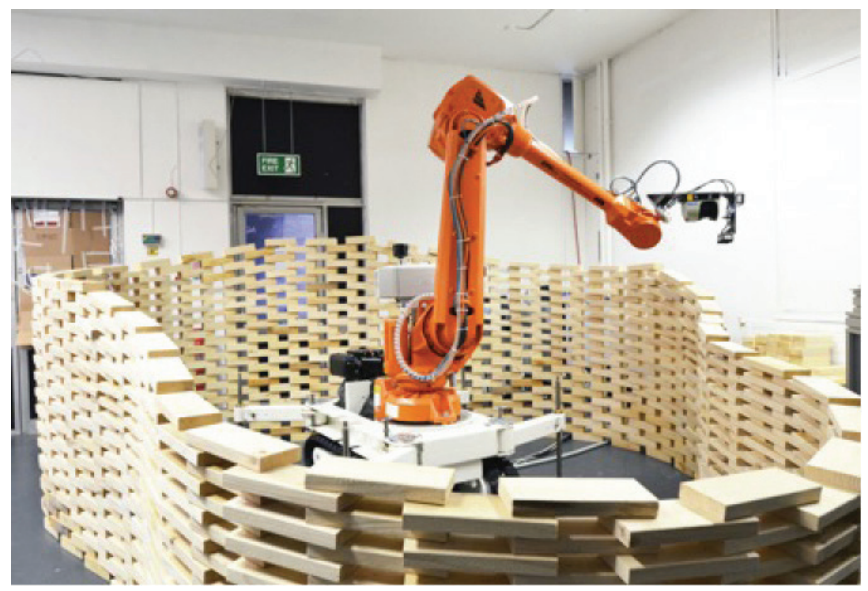

Figure 1: Stratifications, Gramazio \& Kohler, Robotic Fabrication Laboratory, ETH Zurich, 2011.

\section{Background}

Research into on-site robotic assembly has been an area of study since the 1990s (Helm et al., 2014). The ROCCO (Andres et al., 1994), and BRONCO (Pritschow et al., 1996) projects proposed automated assembly methods focusing on solving traditional rationalization problems such as heavy brick lifting and repetitive tasks (Helm et al., 2014). The attempt to introduce robots into the construction industry is not a new research topic, however, few projects involving industrial robots have currently been implemented in the construction industry with the aim of enhancing design-construction processes.

The mobile robotic unit R-O-B, developed by the Gramazio $\&$ Kohler group at the Robotic Fabrication Laboratory (founded in 2005), was the first attempt to implement an entire robotic unit on site for non-standard brick walls. The R-O-B unit was put into operation for the production of Structural Oscillations in 2008 and Pike Loop in 2009 (Gramazio, Kohler \& Willmann, 2014).It includedan industrial robot mounted onalinear axisand housed in a modified freight container which could be moved directly to the construction site (Helm et al., 2014). Although both installations were produced through a curatorial process, closer to an artistic endeavor than a real industrial application, both experimentations were crucial in the development of interest in robotic assembly in the architecture community, showing the expressive potential of automated construction.

The same research group took the next steps in 2012 with the introduction of dimRob, a completely adaptable mobile unit (Helm, 2014). This unit introduced crucial features for on-site construction: feedback and localization techniques for tolerance handling and human-machine interaction (Helm et al., 2014). Using sensor technologies, the environment information is gathered and processed in real time: the robot autonomously recognizes its own position, its context and the actual dimensions of the construction elements on-site. Although these mechanisms were vital steps towards the real application of robots in the construction site, the projects developed by dimRob unit were still focused on non-standard brick layering.
More recently, the Robotic Fabrication Laboratory has expanded its geometric repertoire of robotic assembly projects. In Complex Timber Structures, the dimRob unit investigates the relationship between robot workspace and artifact, assembling individual timber elements in a funnelshaped structure (Gramazio, Kohler \& Willmann, 2014). The overall geometry and size is defined in accordance with the robotic assembly unit, optimizing the use of its maximum envelope space.

The Block Research Group, also at ETH, is carrying out other geometrically challenging projects. These explorations show the difficulties involved in the assembly of freeform structures without the use of scaffolding (Deuss et al., 2014). As Deuss et al. point out, advanced digital calculus of this complex geometries is now receiving a lot of interest from the computers graphics field, but the physical construction of such structures still remains challenging and expensive due to the requirement for extensive formwork during assembly (2014).

One of the main problems in robotic assembly of complex structures is handling material tolerances and controlling overall accuracy. Currently, robotic assembly research projects embed intelligence in high-tech systems and devices. In this project, we introduce an alternative approach: we embed intelligence in the geometric configuration of the structure. To achieve this we used diagrids as a structural system, which we found especially efficient and suitable for robotic assembly of complex structures.

\section{Methodology}

In order to evaluate and ground our proposal to rethink the design-construction workflow while enabling the assembly of complex structures, we tested our methodology on a doublecurved mushroom-shaped structure based on Pier Luigi Nervi's diagrid (Joedicke et al., 1957). A full-scale portion of that structure was fabricated using a 3-axis $\mathrm{CNC}$ machine and assembled by a KUKA KR10 with a customized vacuum tool as end effector for picking and placing components (Figure 2).

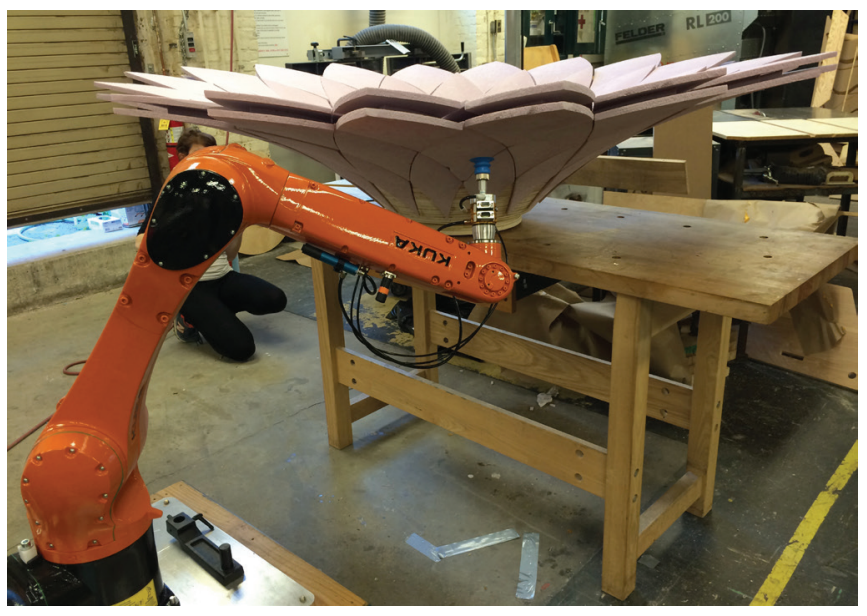

Figure 2: Test assembly of self-supporting double curved structure. 
The robot was attached to a small trolley, to allow its movement and positioning on-site (Figure 3). The project serves us as a case study and a proof of concept that demonstrates the great potential of robotic assembly for complex architectural structures. The following paragraphs describe the development of the project and the methodology we used to create it.

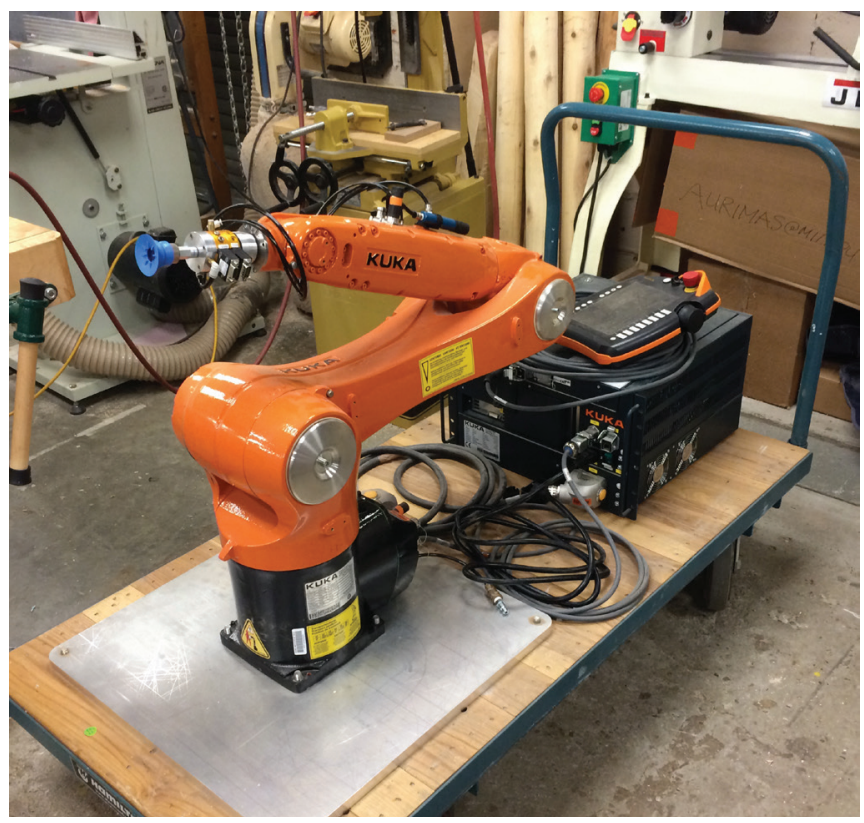

Figure 3: Portable Robot for On-site Assembly. KUKA KR10 attached to a small trolley to allow the movement and positioning of the robot on-site.

\section{Design for Robotic Assembly}

The first step in incorporating the robot into the design process is to understand its abilities and constraints. We therefore performed some iterative tests to evaluate the interaction between the desired designand therobotcapacitiesina robot-programmingenvironment.

The results of these tests led to the adjustment of the overall scale of the structure relative to the maximum reachable point of the robot's envelope space. The structure's dimensions were therefore $1.40 \mathrm{~m} / 4.6 \mathrm{ft}$ tall, 3m/9.8ft wide (Figure 4). In the next step we subdivided the structure into smaller parts in accordance with the diagrid, taking into account the lifting and weight capacity of the robot's end-effector (Figure 5). This discretization can be achieved by any diagrid panelization algorithm.

\section{Structural Scheme and Sequence of Assembly}

Merging two typologies - the tower and the bridge - the subdivided mushroom structure works in pure compression, tightened with a tension ring at the top. In order to connect the subdivided components and to assure structural efficiency, we created a malefemalecantilevered joint(Figure6-8). The result was a self-supporting structure that relies on the continuous compression of each element. In order to enable the smooth sliding of each piece into the other, we made several tests and prototypes at different scales. Those tests helpedustoadjust thegeometry and thetoleranceof themale-female joint and to evaluate the accuracy range of the robot (Figure 9-11). The structure will then be assembled ring by ring, which ensures that it will be self-supported during each of the assembly phases, and omits the use scaffolding (Figure12).

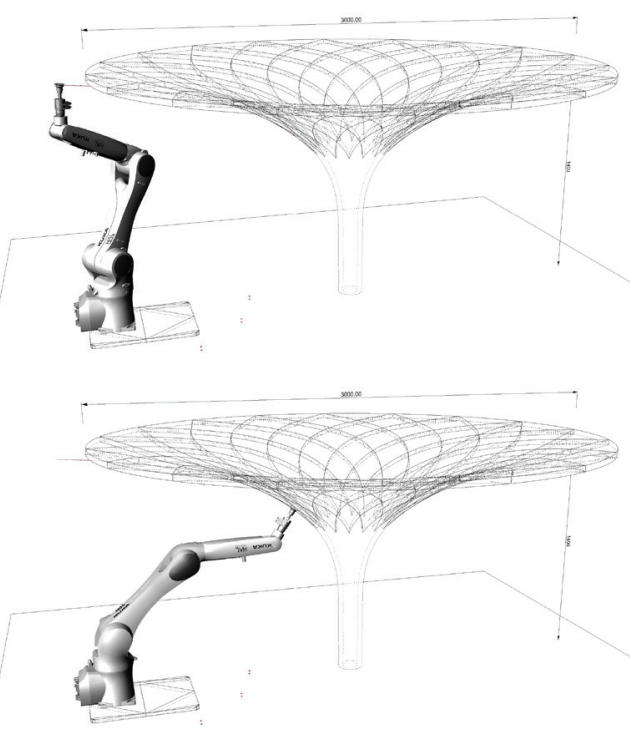

Figure 4: Re-modeling the mushroom structure to meet robot and fabrication constraints. Height: 1.4 meters / 4.6 feet at outer edge. Diameter: 3 meters / 9.8 feet.

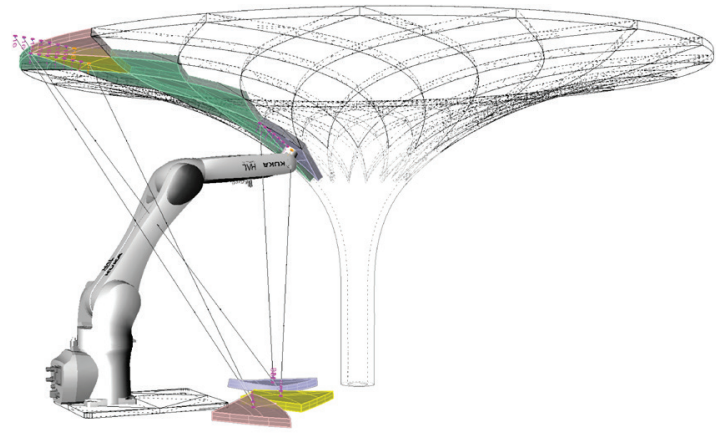

Figure 5: Simulation of assembly in accordance with the maximum reachable points of the robot's envelope space - full scale.

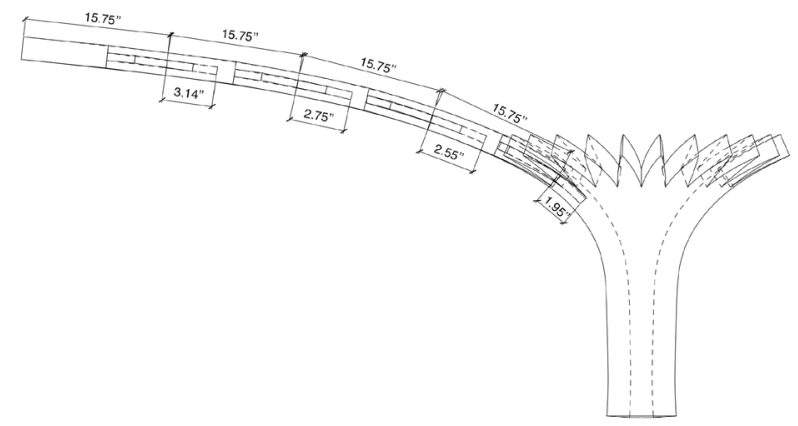

Figure 6: Section of structure: varying offset of cantilevered joint in accordance with the structural scheme. 


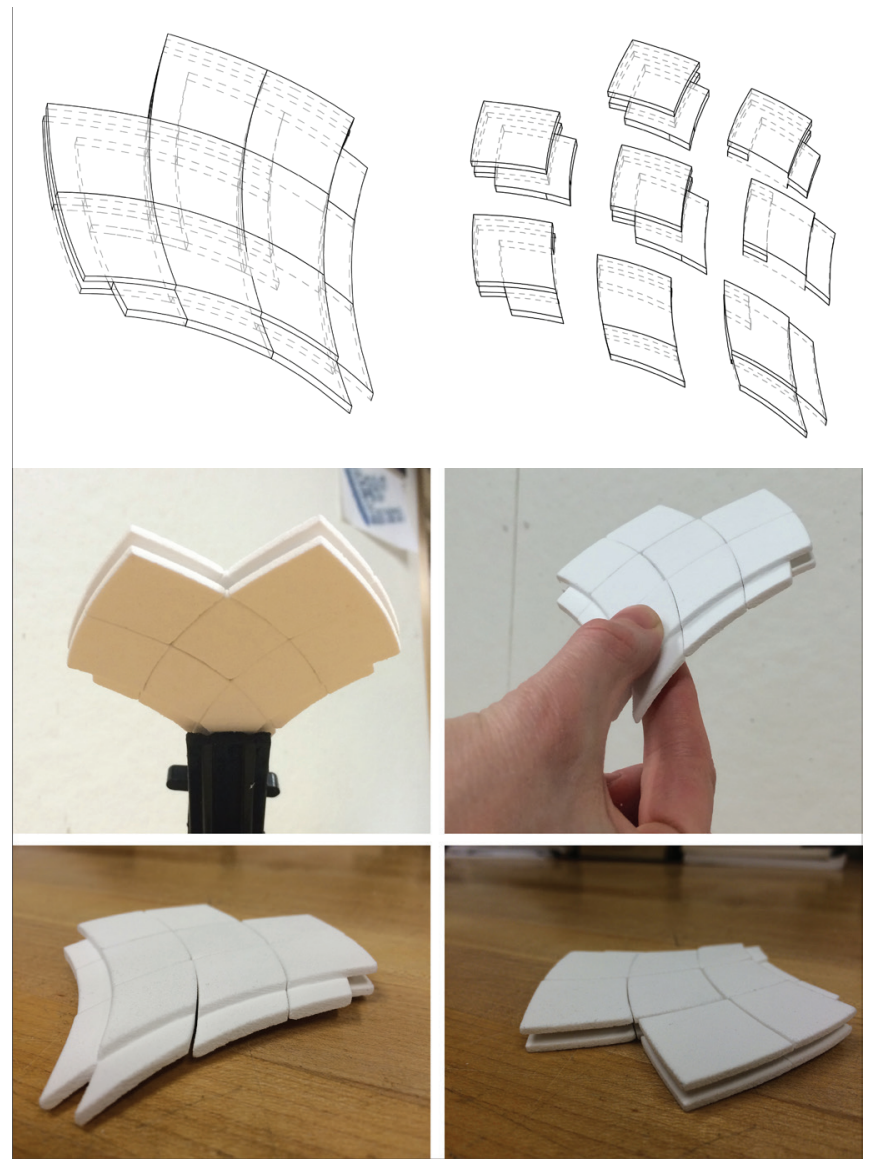

Figure 7-8: Cantilevered components for a stacked self-supporting structure with male/female cantilevered joints. Small-scale prototype, 3D printed in ZCorp.

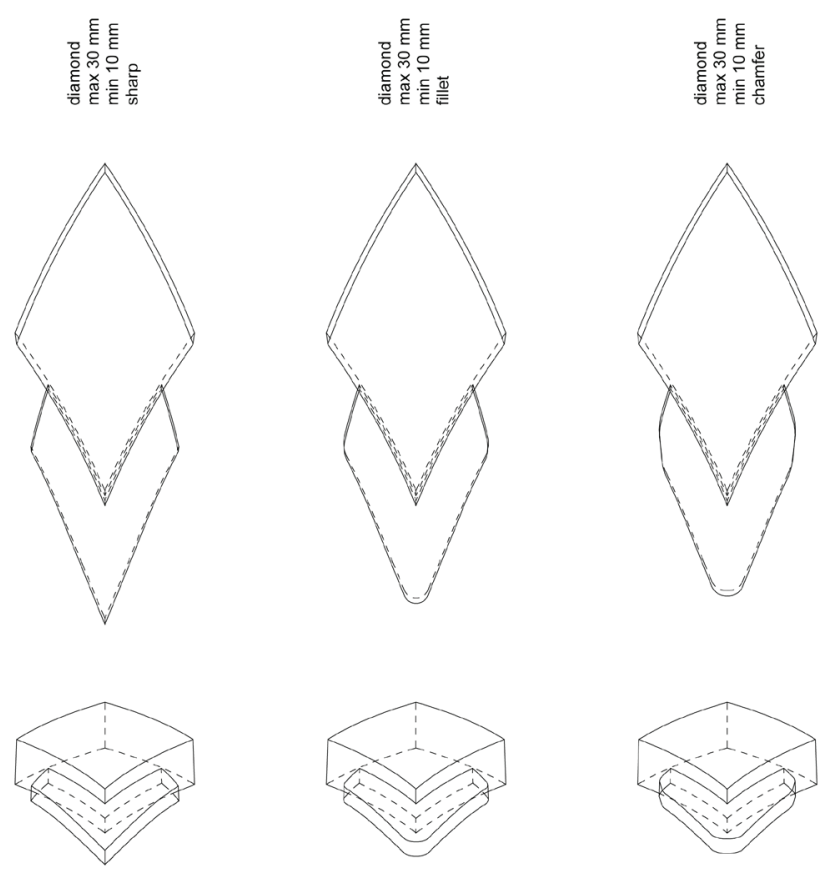

Figure 9: Prototypes for edge tolerance and smoothness tests.

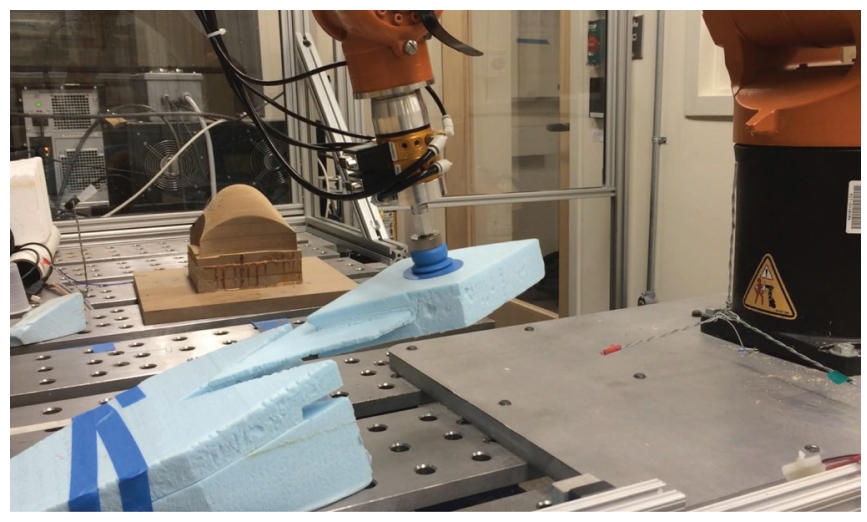

Figure 10: Testing different tolerances with the robot. Medium scale.

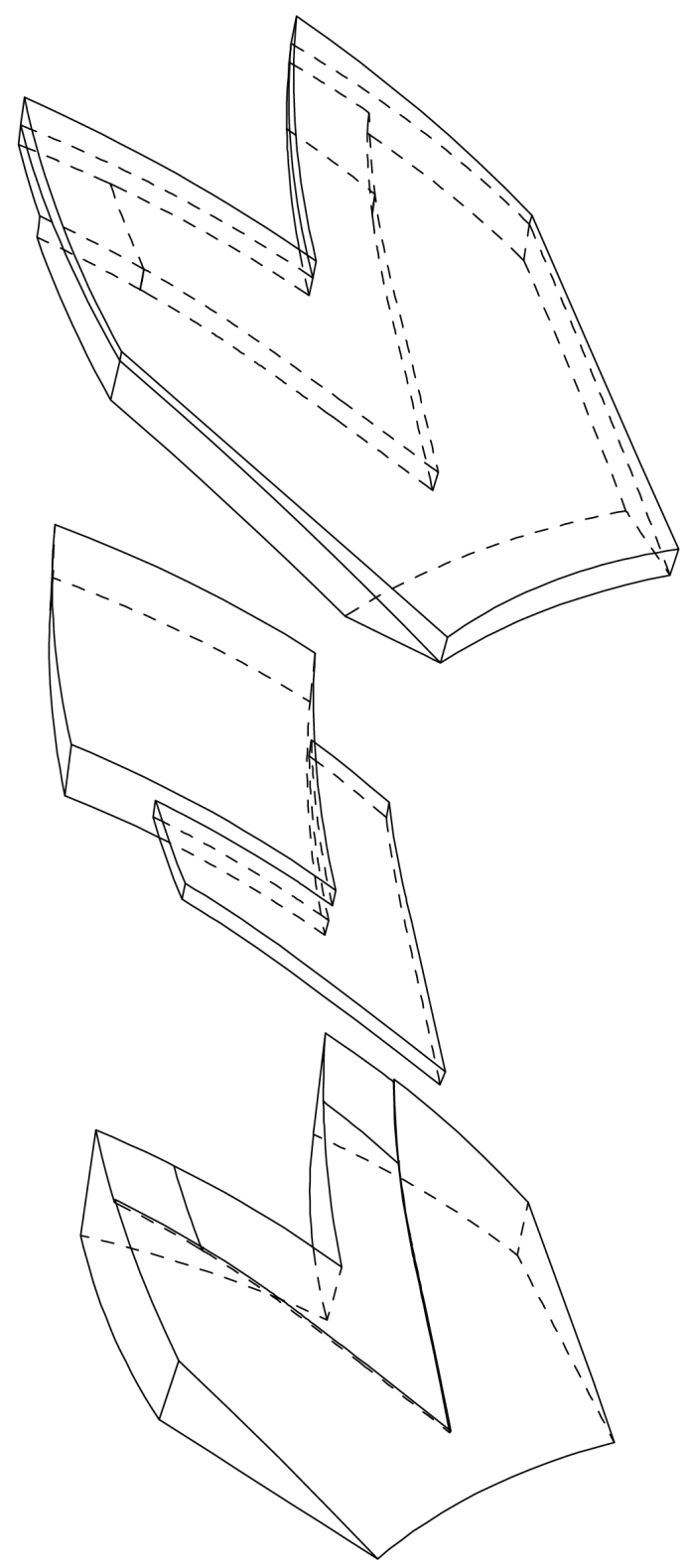

Figure 11: Medium-scale fabrication test: 3 part prototype. 

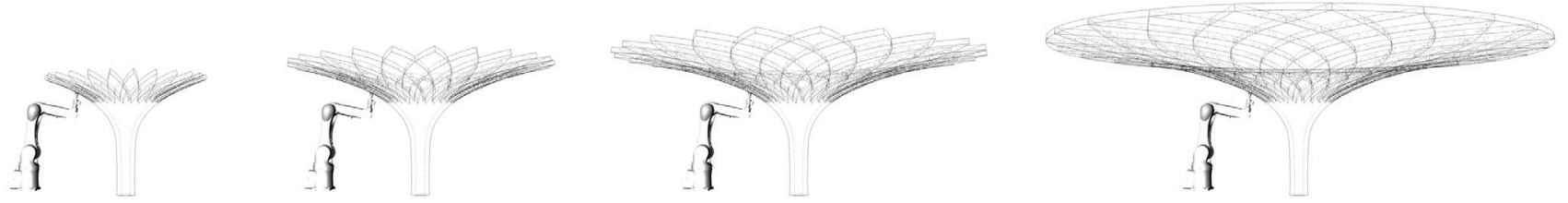

Figure 12: Sequence of Assembly - ring by ring. Structure in compression, edge of structure (final ring) in tension. The structure is self-supported during each of the assembly phases, which omits the use scaffolding.

\section{Method of Assembly}

The next step was to find a suitable toolpath (a description of the robot's movements in space) for the assembly of each piece. We extracted and extended an isocurve from the piece and used it as an input for the programming sequence so that the robot moves (through sequential linear movements) in the same uv planeas that of the given 3D shape (Figure 13-14). This simple matching between the geometric information and the robotic arm toolpath assures a smooth fit and also guarantees no overlapping between neighboring pieces.

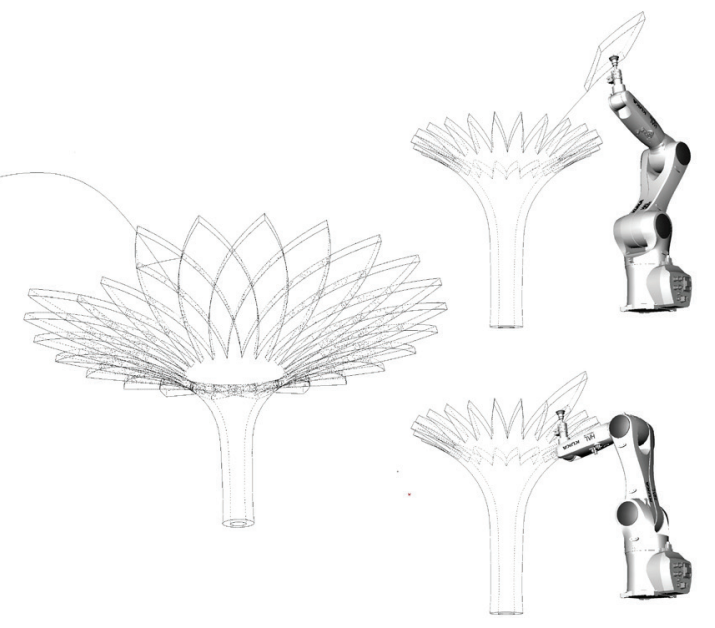

Figure 13: Method of assembly: the central isocurve as the robot's toolpath.

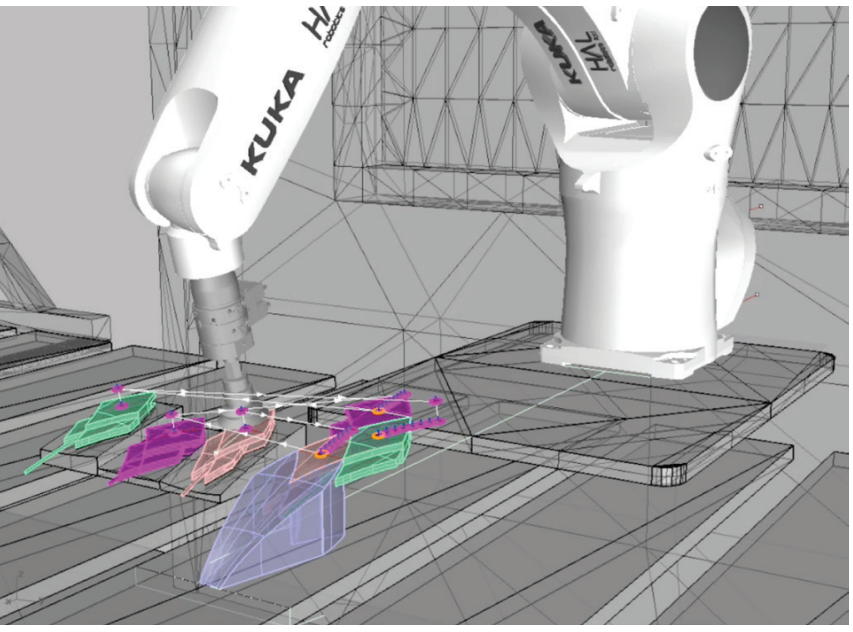

Figure 14: Small-scale simulation. Testing central isocurve and diagrid isocurves as toolpaths.

\section{Materials and Tolerance Handling}

For the full-scale prototype we choose foam as the component's material. Thislightweightmaterialfitswell with thecarrying capacity of the vacuum end-effector we used. The foam is rigid enough to be structural, and at the same time flexible enough to increase tolerance during assembly, in case of inaccuracies. The components were fabricated using a 3-axis $\mathrm{CNC}$ machine. Each component was made of three double curved pieces. Due to the double curvature on both sides of each piece, all the pieces were flip milled (Figure15-16).

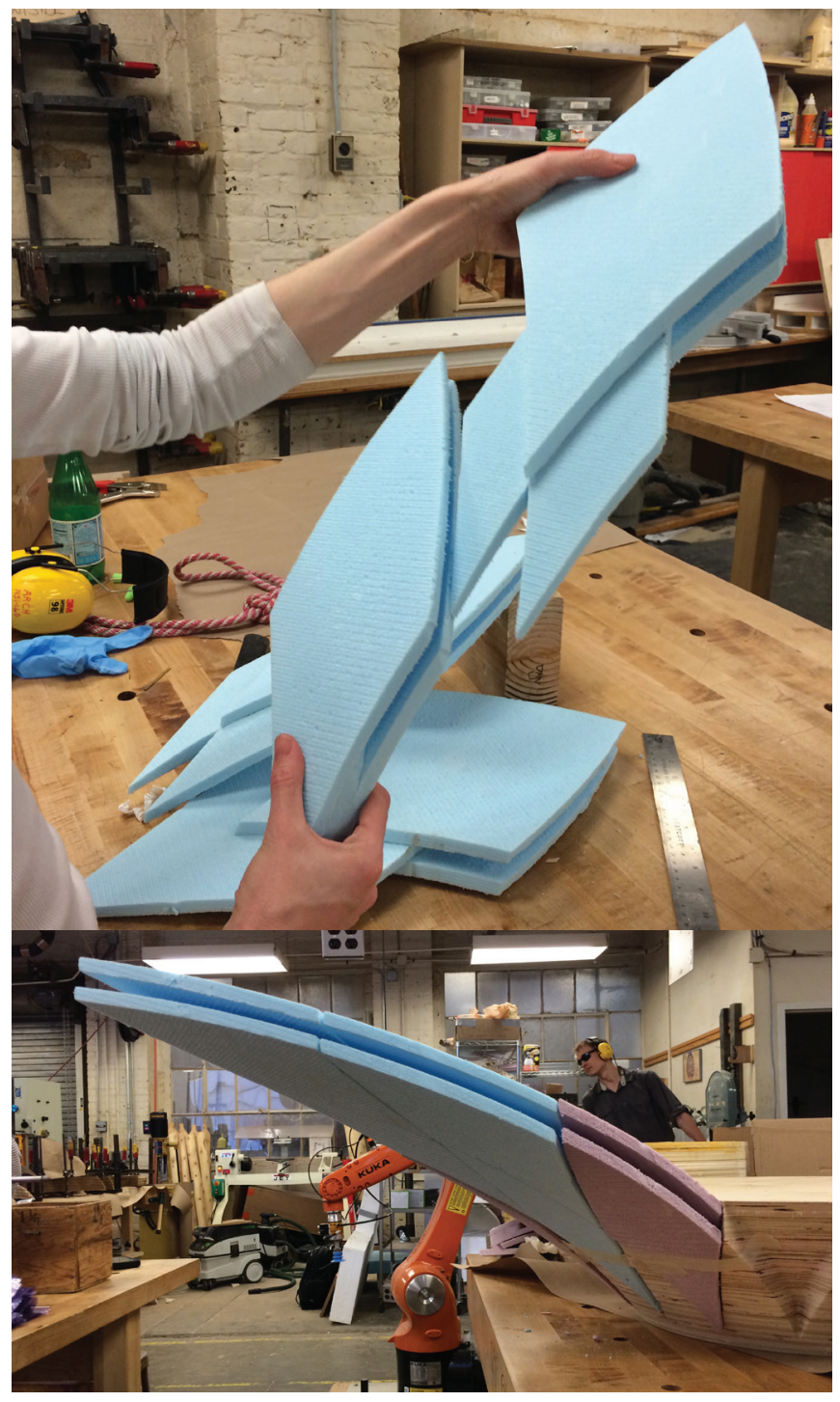

Figure 15-16: Full-scale components, each made of three doublecurved pieces. 
As introduced before, one of the main difficulties in architectural robotic assembly is dealing with tolerances and inaccuracies. The unique geometry of the cantilevered components has a crucial role in tackling tolerance issues and gaps between the digital model and the actual object. It guides the correct assembly of each piece: the assembled component needs only to slide slightly into the target component in order for it to register and interlock in the correct position, even if the initial position was inaccurate. Thus, the combination of the unique geometry and the material properties offers a powerful solution for tolerance issues.

\section{On-Site Assembly}

In order to assemble the structure on-site, we attached a KUKA KR10 to a small trolley to allow the movement and positioning of the robot. Once the structure's location was decided, the location of the robot was fixed during the whole process of assembly. In order to assemble the whole structure we developed a simple and accurate rotation system by attaching a rotary plate with a fixed rotation angle of 60 degrees to the bottom of the column. The 60-degree angle was chosen in accordance with the maximum reachable points of the robot's envelope space. So the code for assembly was set to perform the assembly of 1/6th of each ring at a time, and was repeated 6 times so as to complete a full circumference (Figure 17-18). Each component to be assembled was positioned parallel to the ground, and during its movement to the target location it was rotated in accordance with the extracted central isocurve that was used as the toolpath (see 3.3 Method of assembly). The components were piled on top of each other, a separate pile for each ring.

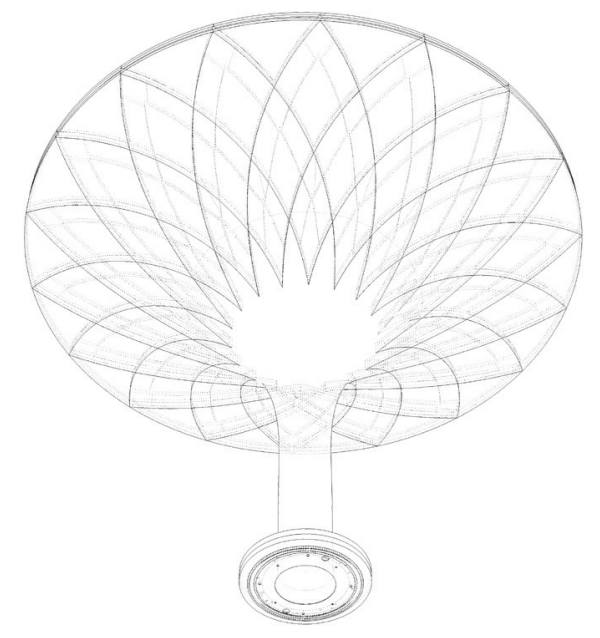

Figure 17: A portion of the full structure with the rotary plate at the bottom.

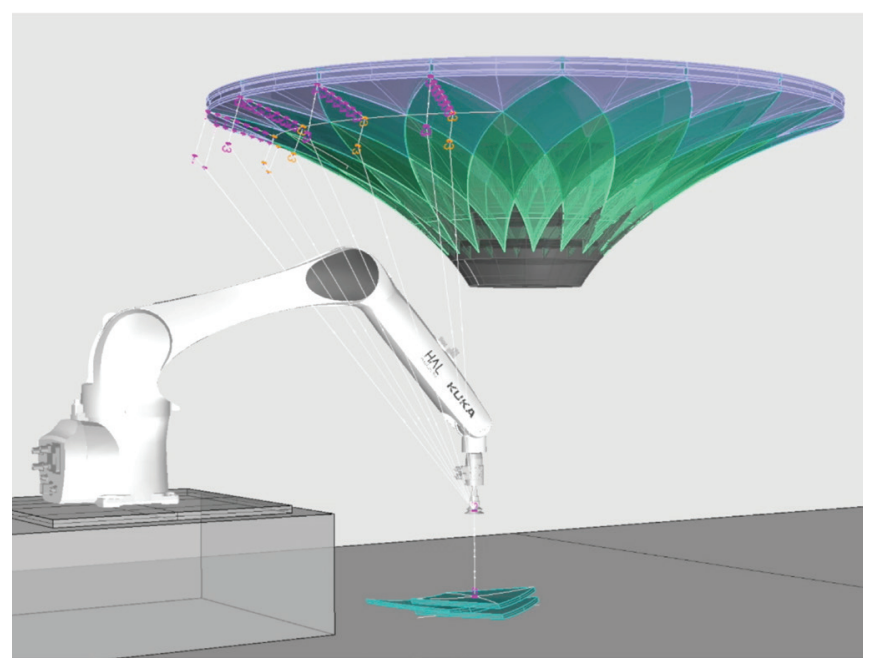

Figure 18: A full-scale simulation of 1/6th of the structure to be assembled using the component's central isocurve as a toolpath.

\section{Results}

As a proof of concept for an approach to an on-site robotic assembly of complex structures, we fabricated a portion of a $1.40 \mathrm{~m} / 4.6 \mathrm{ft}$ tall, $3 \mathrm{~m} / 9.8 \mathrm{ft}$ wide self-supporting structure that was assembled on-site by a KUKA KR10 (Figure 19).

As part of the process we developed a unique geometry of components with a male-female cantilevered joint that is suitable for the assembly of any thin double curved structure and can be easily fabricated with any 3-axis CNC machine. This unique geometry helps to solve issues of tolerance and inaccuracy that inevitably occur in fabrication and on-site construction processes. Additionally, we developed a code that translates the geometrical information of any diagridbased structure into toolpaths for robotic assembly.

\section{Conclusions}

\section{Contributions}

In this project we:

- Implemented a method for translating the structure's geometric information into inputs in the robot code. These inputs serve as the robot's toolpath for pick and place assembly.

- Demonstrated that the assembly of complex structures can be as easy as generating these structures on the computer.

\section{Future Work}

In order to further develop robotic assembly for complex architectural structures, and in order to have the ability to 

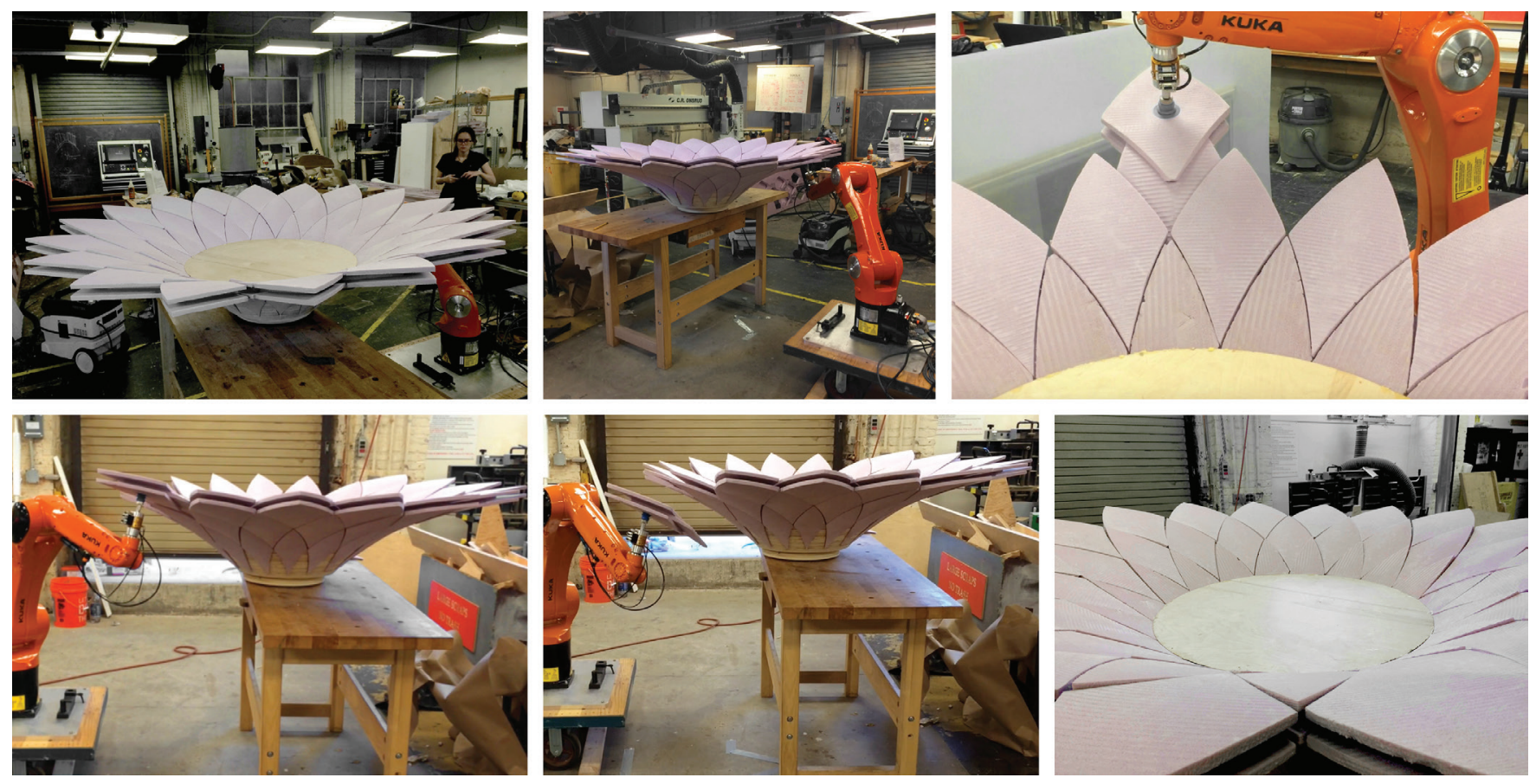

Figure 19: Full-scale assembly of final prototype.

incorporate robots into the design-construction workflow, a few steps need to be considered:

- Tolerance handling: As already mentioned, the combination of the unique geometry of the cantilevered components and their material properties, offers a powerful solution to tolerance issues. Nevertheless, in order to achieve even greater accuracy in real-time and to allow the use of more rigid and less flexible materials, a feedback and localization system could be considered (Kahane \& Rosenfeld, 2004).

- From proof of concept to full/large scale structures: Current studies in robotic architectural assembly usually have their results in the form of pavilions or temporary installations (Gramazio, Kohler \& Willmann, 2014). The unique features of the mushroom column allow it to perform as a customized yet standard building component. Thus, these features can be easily implemented in any type of building, and therefore holds a potential for robotic assembly in large-scale structures. In order to translate the aforementioned unique properties of the foam (rigid, yet flexible and lightweight) into a suitable construction material we suggest examining the use of lightweight composites. This material combines load-bearing capacity with durability. Taking current digital fabrication possibilities into consideration, it stands out as a manageable casting material (Costanza, 2015).

- Applying the method to other complex structures: Interesting future work may include testing the proposed method in other types of diagrid-based structures. We believe that the proposed methodology can be also applicable to freeform and asymmetrical structures. By discretizing those structures into diagrid components that contain the cantilevered joint presented in this paper, the structure could be assembled without scaffolding, being in compression during assembly, and tightened at the end of assembly in order to reinforce it.

\section{Acknowledgements}

This project was developed as part of the class "(Re) fabricating Tectonic Prototypes" at Harvard University Graduate School of Design, during Spring 2015. Instructor: Leire Asensio-Villoria. Structural Consultant: Hanif Kara. We used the HAL plugin for Grasshopper developed by Thibault Schwartz in order to simulate the assembly process and to generate the initial code. We would like to thank Justin Lavallee, director of MIT Architecture Shop and Christopher Dewart, manager of the MIT Architecture Woodshop, for their support and valuable advice. We would also like to thank Caitlin Mueller for her insightful comments.

\section{References}

Carlsson, B. (1989). "The evolution of manufacturing technology and its impact on industrial structure: an international study". Small Business Economics, 1(1), 21-37.

Costanza D. Fibrous Tectonics [e-book]. 2015. Available from: MIT Barton Catalog, Ipswich, MA.

Deuss M., Panozzo D., Whiting E., Liu Y., Block P., HornungSorkine O. and Pauly M. "Assembling Self-Supporting Structures", ACM Transactions on Graphics - SIGGRAPH Asia 2014,33(6): 214:1-214:10, 2014.

Gambao, E., Balaguer, C., \& Gebhart, F. (2000). Robot assembly system for computer-integrated construction. Automation In Construction, 9479-487.

Gramazio, Fabio, Matthias Kohler, and Jan Willmann. The Robotic Touch: How Robots Change Architecture. Zurich: Park Books, 2014. 
Helm, V. (2014), "In-Situ Fabrication: Mobile Robotic Units on Construction Sites”. Archit Design, 84:100-107.

Helm, V., Willmann, J., Gramazio, F., \& Kohler, M. (2014). "InSitu Robotic Fabrication: Advanced Digital Manufacturing Beyond the Laboratory. Gearing Up \& Accelerating CrossFertilization Between Academic \& Industrial Robotics Research In Europe", 63.

J. Andres, T. Bock, F. Gebhart, and W. Steck, "First results of the development of the masonry robot system ROCCO," in Proc. 11th ISARC International Symposium on Automation and Robotics in Construction, Brighton, 1994, pp. 87-93.

Kahane, B., \& Rosenfeld, Y. (2004). "Real-time 'Sense-andAct' operation for construction robots". Automation In Construction, 13(6), 751-764.

Nervi, P. L. (1957). The works of Pier Luigi Nervi. New York, F.A. Praeger [1957].

Pritschow, G., Dalacker, M., Kurz, J., \& Gaenssle, M. (1996). "Technological aspects in the development of a mobile bricklaying robot". Automation In Construction, 5(1), 3-13. 